\title{
Heel Pressure Injuries: Consensus-Based Recommendations for Assessment and Management
}

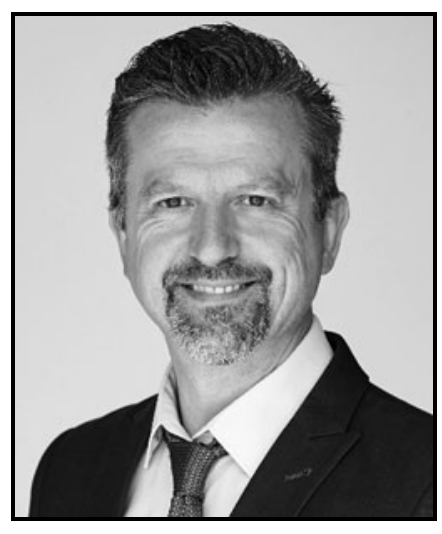

Massimo Rivolo, RN, RGN, BSc (Hons), MSc Wound Care

Submitted for publication June 27, 2019. Accepted in revised form September 12, 2019.

${ }^{*}$ Correspondence: Independent Tissue Viability Nurse Consultant, Turin 10060, Italy. (e-mail: massimo.rivolo@gmail.com).

\author{
Massimo Rivolo, ${ }^{1, *}$ Sara Dionisi, ${ }^{2}$ Diletta Olivari, ${ }^{3}$ Guido Ciprandi, ${ }^{4}$ \\ Serena Crucianelli, ${ }^{4}$ Silvia Marcadelli, ${ }^{2}$ Rosa Rita Zortea, \\ Fabio Bellini, ${ }^{6}$ Matteo Martinato, ${ }^{7}$ Armando Gabrielli, ${ }_{8}^{3}$ \\ and Giovanni Pomponio \\ ${ }^{1}$ Independent Tissue Viability Nurse Consultant, Turin, Italy. \\ ${ }^{2}$ AUSL Bologna, Bologna, Italy. \\ ${ }^{3}$ Clinica Medica, Università Politecnica delle Marche, Ancona, Italy. \\ ${ }^{4}$ Ospedale Pediatrico Bambin Gesu', Roma, Italy. \\ ${ }^{5}$ Turriaco GO, Turriaco, Italy \\ ${ }^{6}$ ATS Val Camonica, Sondrio, Italy. \\ ${ }^{7}$ Azienda Ospedaliera di Padova, Padova, Italy \\ ${ }^{8}$ Clinica Medica, Ospedali Riuniti di Ancona, Ancona, Italy.
}

Significance: A systematic approach to develop experts-based recommendations could have a favorable impact on clinical problems characterized by scarce and low-quality evidence as heel pressure ulcers.

Recent Advances: A systematic approach was used to conduce a formal consensus initiative. A multidisciplinary panel of experts identified relevant clinical questions, performed a systematic search of the literature, and created a list of statements. GRADE Working Group guidelines were followed. An independent international jury reviewed and voted recommendations for clinical practice. Consent was developed according to Delphi rules and GRADE method was used to attribute grade of strength.

Critical Issues: The extensive search of the literature retrieved 42 pertinent articles (26 clinical studies, 7 systematic reviews or meta-analysis, 5 other reviews, 2 consensus-based articles, and 2 in vitro studies). Thirty-five recommendations and statements were created. Only 1 of 35, concerning ankle-brachial pressure index reliability in diabetic patients, was rejected by the panel. No sufficient agreement was achieved on toe brachial index test to rule out the orphan heel syndrome, removing dry eschar in adult patients without vascular impairment, and using an antimicrobial dressing in children with infected heel pressure injuries. Eleven recommendations were approved with a weak grade of strength. Experts strongly endorsed 20 recommendations. Offloading, stages I and II pressure injuries, and referral criteria were areas characterized by higher level of agreement.

Future Directions: We believe that the results of our effort could improve practice, especially in areas where clear and shared opinions emerged. Barriers and limits that could hinder implementation are also discussed in the article.

Keywords: heel pressure ulcers, guideline, consensus, neonatal/pediatric, diabetic, vascular assessment

(C) Massimo Rivolo, et al. 2019; Published by Mary Ann Liebert, Inc. This Open Access article is distributed under the terms of the Creative Commons Attribution Noncommercial License (http:// creativecommons.org/licenses/by-nc/4.0/) which permits any noncommercial use, distribution, and reproduction in any medium, provided the original author(s) and the source are cited. 


\section{SCOPE AND SIGNIFICANCE}

RECENT DATA FROM U.S. SURVEYS show a slight decline in prevalence of pressure ulcers in the latest 10 years, mostly in acute and rehabilitation care settings, but clinical and organizational burden is still impressive. ${ }^{1-3}$

The heel is the second most common anatomical site for pressure injury following the sacral area in all considered ages. ${ }^{4}$ Prevalence of heel pressure injuries (HPIs) varies from $7.3 \%$ to $18.2 \%^{6}$ and a recent root cause analysis showed a prevalence rate of $51 \%$ of grades III and IV HPIs in the community. ${ }^{7}$

\section{TRANSLATIONAL RELEVANCE}

Despite being so common, causing high risks for patients, HPIs pathophysiology is not yet completely understood. In particular, mechanisms underlying relationship between bed rest and development of heel ulcers still need clarification, ${ }^{8}$ precluding so far the development of adequate strategies for diagnosis and management. Not to mention the importance of the crucial involvement or vital structures such as bone and calcaneal tendon. This is much more evident and disabling in frail elderly and babies. ${ }^{9}$

\section{CLINICAL RELEVANCE}

Currently, there is no standardized ways of early assessing and treating patients with HPI. Lack of well-conducted clinical studies prevents the definition of a shared policy of clinical management and contributes to unjustified heterogeneity in clinical behaviors and patients outcomes.

In particular, clinical evidence is limited to few case series, ${ }^{10}$ largely insufficient to guide practice. On the contrary, a number of narrative reviews are available, ${ }^{11-16}$ showing different and sometimes conflicting opinions among experts coming from different specialties, professions, and countries.

\section{BACKGROUND}

In September 2017, Italian nursing society for wound care study (AISLeC), Italian Nurses' Association for Wound Care, started a project aimed to integrate available poor evidence with experts' opinions and stakeholders' values, to achieve an adequate degree of agreement on recommendations for clinical practice.

To meet this purpose, a formal consensus conference was conducted. This method allows avoiding main biases of informal consensus development methods, as dominance of eminence- or vehemencedriven positions and/or "political" interferences. Moreover, the use of a systematic approach enables to measure agreement, distinguishing those be- haviors that could be part of a good clinical practice from areas of absolute uncertainty.

Our study led to development of recommendations/statements in six specific areas: vascular assessment, management of HPIs stages I and II, management of HPIs stages III and IV lesions suspected deep tissue injuries (STDIs) or injuries of unknown depth (DU), referral criteria, offloading devices, and biophysical agents.

\section{METHODS}

This project was developed and implemented by AISLeC. In early 2017, it set up a steering committee to provide methodological expertise and organizational support, with the aim to create expert-based recommendations on assessment and treatment of HPIs, supporting clinicians in best practice, improving appropriateness of care, and reducing associated costs.

Based on U.S. National Institute of Health (NIH) guidelines, several authors published articles addressing the conduction of formal consensus initiatives. ${ }^{17}$ In our project, this systematic approach, also recommended by the Italian National System for Guidelines, ${ }^{18}$ was used.

The steering committee selected and brought together a multidisciplinary and multiprofessional group of 12 experts forming an advisory panel (Appendix 1).

Members came from the following areas of expertise: nine clinicians involved in wound care (seven nurses, one doctor expert in diabetic foot ulcers, and one surgeon expert in diabetic foot ulcers) and three trained in trial design and statistics (two methodologists nurses and one methodologist doctor).

\section{Phase 1: problem definition, questions building, literature search, and recommendations writing}

Expert panel broke down the clinical problem into six areas of interest to be discussed in a structured way: vascular assessment of the lower limb in the presence of HPIs; assessment and local treatment of HPIs stages I and II; assessment and local treatment of HPIs stages III and IV, DU, and SDTI; referral criteria to address patients to specialized centers; use of biophysical agents in recalcitrant ulcers; and offloading devices in walking and nonwalking patients.

Within these areas, three specific populations were identified: adult, diabetic, and neonatal/pediatric.

For each area, advisory panel defined specific clinical-organizational scenarios and corresponding queries, then methodologists formulated research questions according to EPICOT+ method. ${ }^{19}$ Questions and outcomes were voted for relevance. 
An extended search of the literature was carried out, to assess the current state of evidence on the topic. Three bibliographic databases (PubMed, Scopus, and CINHAL) were searched using a sensitive strategy. In detail, the approved final list of questions and search strings are displayed in Supplementary Table S1.

Retrieved articles were evaluated for pertinence and quality. Clinical studies reporting data from at least one patient were submitted to methodologists for critical appraisal and data extraction.

Evidence summaries and approved questions were debated, leading to the drafting of a preliminary list of statements and recommendations.

The list was thoroughly debated in a plenary meeting (September 2018, Bologna, Italy) through a structured discussion.

Twenty-nine experts (Appendix 2), from three professions (medical, nursing, and podiatric) and five specialties (diabetology, vascular surgery, plastic surgery, dermatology, and pediatric), examined evidence and recommendations together with the whole panel to improve wording, resolve ambiguities, remove futile or potentially harmful statements, and give comments and criticisms.

A final list of 35 statements and recommendations was set up accordingly.

Recommendations/statements were written pursuing the goal of making text clear and unambiguous. Notes including information about limitations and conditions of applicability, details on target population, interventions, settings, and outcomes were also added if necessary.

Recommendations/statements were submitted to jury vote together with related notes.

\section{Phase 2: jury voting process and strength of recommendations attribution}

A multiprofessional and multidisciplinary international 16-member jury was established (Appendix 3). Acknowledged specific clinical and scientific expertise on heel lesions was the main selection criterion.

GRADE method ${ }^{20}$ was followed to build absentee ballots, interpreter jurors' votes, and attribute strength to recommendations and statements.

In particular, a scale ranging from 0 (absolutely not recommended/approved) to 9 (strongly recommended/approved) was used. Interquartile ranges (IQRs) and medians were calculated to assess the level of agreement. ${ }^{21} \mathrm{~A}$ recommendation or statement was defined as being:

- "Strongly recommended/approved" if the median was $\geq 8$ and the lower level of the IQR was $>5$.
- "Weakly recommended/approved" if the median was 6 or 7 and the lower boundary of the $\mathrm{IQR}$ was $\geq 5$.

- "Not recommended/approved" in the case when median was $<5$ and the upper boundary of $\mathrm{IQR}$ was $\leq 5$.

- "Uncertain" in the remaining situations (median $=5$; median $>5$ but lower quartile $<5$; median $<5$ but upper quartile $>5$ ).

The percentage of "strong agreement" was also calculated and reported in Results session.

Each statement and recommendation, with related evidence summary and specific comments, was written in the ballots in a structured format and from a neutral point of view.

Delphi method was used to develop and measure agreement among jurors. A two-round strategy was chosen.

In particular, in the first round, jury members were asked through email to vote and provide eventual further suggestions about statements wording or notes.

Then, in a second round, each juror could compare his or her own opinion with others' opinion (provided in an anonymous way), before giving a second, definitive, vote.

\section{DISCUSSION OF FINDINGS AND RELEVANT LITERATURE}

The extensive search of the literature followed by a careful manual screening of retrieved articles led to find 42 articles pertinent to our 34 questions. Search algorithm is detailed in Supplementary Fig. S1.

Supplementary Table S2 summarizes main characteristics, results, methodological comments, and level of evidence for 26 clinical studies. In addition, seven systematic documents (five systematic reviews, ${ }^{16,22-25}$ one report of technology assessment, ${ }^{26}$ and one meta-analysis ${ }^{27}$ ), two in vitro studies, ${ }^{28,29}$ four narrative reviews, ${ }^{13,30-32}$ and two consensusbased articles ${ }^{33,34}$ were summarized in structured format and made available to experts.

Overall, only 1 of 35 recommendations, concerning ankle-brachial pressure index (ABPI) reliability in diabetic patients, was rejected by the panel, whereas no sufficient agreement was achieved to recommend in favor or against performing toe brachial index test to rule out the orphan heel syndrome (OHS), removing dry eschar in adult patients without vascular impairment, and using an antimicrobial dressing in neonates and pediatric patients with infected HPIs.

Eleven recommendations were approved with a weak grade of strength, mainly in "HPIs III and IV SDTI and DU" and "biophysical agents" areas. 
Experts strongly endorsed 20 recommendations. "Offloading," "HPIs I and II stages," and "referral criteria" were the areas characterized by higher level of agreement.

\section{Vascular assessment}

Text of recommendations and statements, notes, and voting results are listed in Table 1.

RECOMMENDATION 1: In all diabetic and nondiabetic adults presenting with one or more HPIs, a manual ABPI test including the peroneal artery should be performed to evaluate a limb ischemia. Grade: Weakly recommended.

STATEMENT 1: In all adults and diabetic patients presenting with HPIs, a full vascular assessment of the lower limb requires an ABPI performed on each artery (posterior tibial artery [PTA], dorsalis pedis, and peroneal) Grade: Weak agreement.

ABPI is the first noninvasive test to evaluate vascular supply in the lower limbs. ${ }^{15} \mathrm{ABPI}$ is usually performed using the PTA and pedis artery, but it may not provide a full picture on the rear foot perfusion. ${ }^{35}$ The panel was interested in understanding whether adding the peroneal artery, beyond carrying out selective ABPIs on each other artery, makes the test more reliable in detecting arterial insufficiency in routine practice, as also postulated in angiosomes theory.

The only relevant article,${ }^{35}$ among those pertinent to this question, ${ }^{36-38}$ underlines the relevance of peroneal artery in supplying blood to the lateral part of the heel.

Nowadays, in common practice, manual ABPI performed using tibial arteries only (posterior and anterior) could not provide information regarding the hind foot (perfusion). In one retrospective study, ${ }^{35} 50 \%$ of ABPI was performed using the anterior tibial artery only, making the vascular assessment even less reliable in the case of HPIs.

Selective ABPI tests, separately performed on the three arteries, could be a more complete tool, useful in ruling out the suspicion of the OHS.

During the meeting held in Bologna in September 2018, experts confirmed the importance of PTA assessment for heel perfusion evaluation as the main important artery for the heel vascular supply as well as the value of including a selective ABPI in routinary practice.

The jury confirmed the recommendation, even if with a weak ranking.

As performing this test requires skilled professionals and could be time consuming, implementation of this recommendation on a daily basis could be difficult in peripheral not specialized settings, especially for district nurses.
Using Doppler ultrasound or more advanced diagnostic tests could provide a better picture over ABPI in the perfusion of the foot, in particular of the hind foot. However, this intervention was not specifically discussed in our project, due to its limited availability in primary clinical settings. Specific research on this topic is strongly desirable.

Moreover, the panel agreed that automatic ABPI in evaluating the peroneal artery is not appropriate.

Finally, it is advisable to underline that the ABPI has some limits per se, and probably it is not an accurate test in case of limb ischemia, due to its low reliability. ${ }^{39}$

In an ancillary statement, we asked jurors to vote about the need of performing an ABPI on each artery to define a complete vascular assessment of lower limb. A weak agreement was achieved.

STATEMENT 2: In diabetic patients with one or more HPIs, an ABPI is a reliable test. Grade: Rejected.

A growing body of evidence shows the lack of validity of the ABPI test in diabetic patients, due to high prevalence of artery wall calcification and stage III chronic kidney disease. ${ }^{40}$ Moreover, in this population, ABPI showed poor ability in detecting severe stenosis $(>50 \%$ to $>75 \%$ ), when compared with reference standard. The jury endorsed this point of view rejecting the statement.

Anyway, the same guidance from National Institute for Clinical Excellence showed conflicting evidence of efficacy also for toe brachial pressure index (TBPI), waveform analysis, and pulse oximetry. Small studies seem to attribute promising results to the latter two interventions (mainly for the waveform analysis), but future clinical trials are needed.

RECOMMENDATION 3: In all diabetic and nondiabetic adults presenting with one or more HPIs, jurors cannot recommend in favor or against performing a TBPI test to rule out the OHS. Grade: Uncertain. Not approved.

One important topic debated by the panel was the use of TBPI in completing a vascular assessment. The question addresses the utility of TBPI in assessing the hind foot perfusion. According to angiosomes theory, the lower limb is characterized by vascular patterns, perfused by specific arteries or parts of them. Calcaneal branches of the posterior tibial and peroneal arteries are far from the big toe where arterial pressure is usually measured.

Some studies suggest that adding TBPI to the standard test, such as ABPI, could complete and make more accurate the vascular assessment, due to its better sensitivity. ${ }^{41}$

However, opinions among experts either involved in our panel or in the jury were discordant. 
Table 1. Notes (as voted by the jury) associated with recommendations and statements, results of voting process and level of strength attributed according to the GRADE method

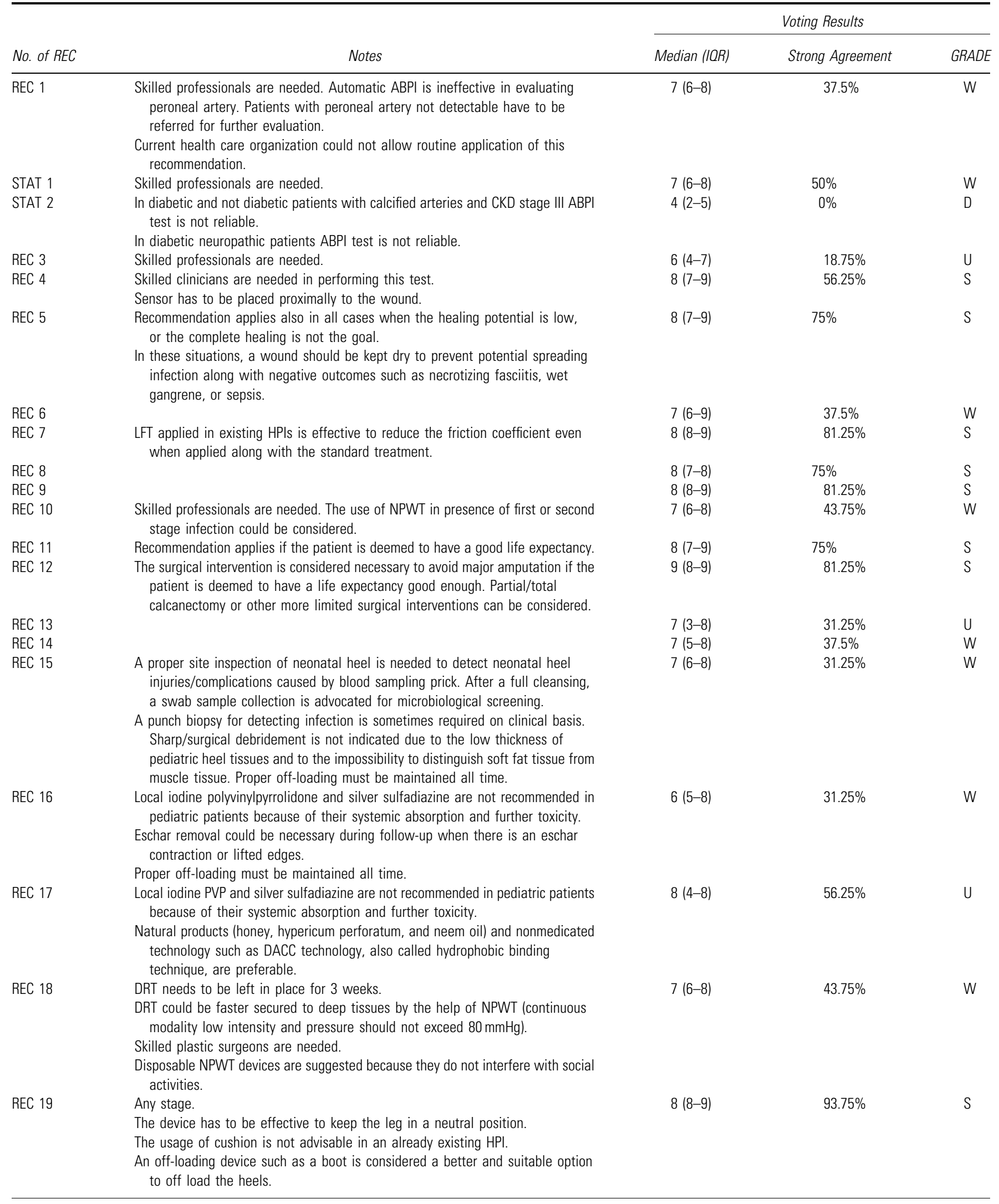


Table 1. (Continued)

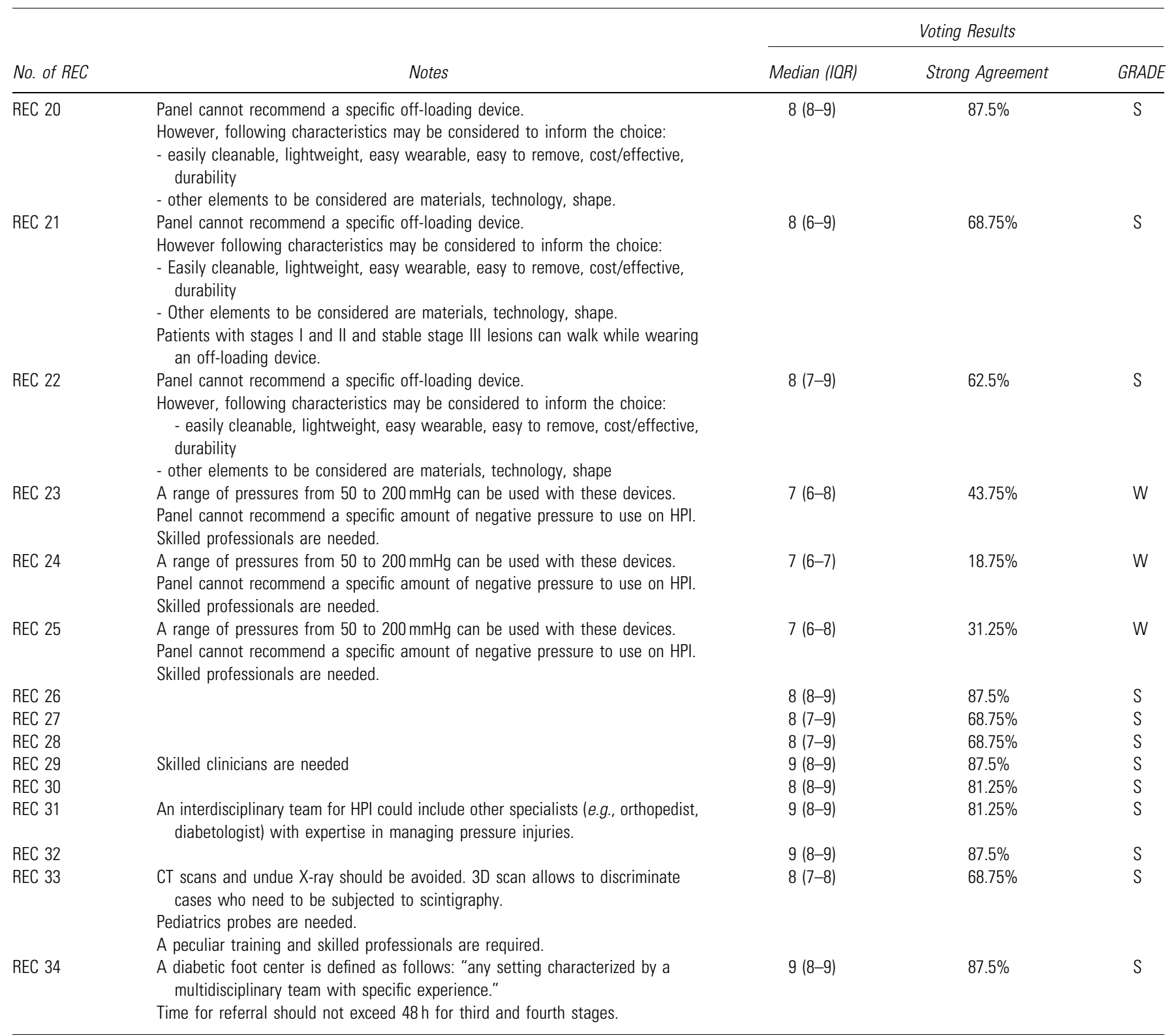

For the text of recommendations and statements and details about voting interpretation, see Results and Methods sections, respectively.

3D, three-dimensional; ABPI, ankle-brachial pressure index; CKD, chronic kidney disease; CT, computed tomography; DACC, dialkylcarbamoyl chloride; DRT, dermal regeneration template; HPIs, heel pressure injuries; IQR, interquartile range; LFT, low friction technology; NPWT, negative pressure wound therapy; PVP, polyvinylpyrrolidone; S, strongly recommended; U, uncertain; W, weakly recommended

Therefore, we cannot suggest the routine use of TBPI in clinical practice in presence of HPIs.

The preliminary data on diagnostic accuracy of TBPI in patients with HPIs should stimulate further clinical research to evaluate its appropriateness in hind foot vascular assessment.

RECOMMENDATION 4: In diabetic and nondiabetic adults presenting with one or more HPIs, the transcutaneous oxygen pressure $\left(\mathrm{TcPO}_{2}\right)$, with sensors applied on the hind foot, must be performed to assess a calcaneal ischemia and rule out the OHS. Grade: Strongly recommended.
The OHS results in absent circulation with a normal fore foot perfusion. Few articles about the OHS have been published ${ }^{42,43}$ mainly in diabetic population, showing how the heel can be ischemic despite a normal fore foot perfusion.

The panel was interested in defining how to best assess the arterial supply of the lower limb with HPI, given the already discussed ABPI limits, ${ }^{39}$ especially affecting the evaluation of hind foot in diabetic and nondiabetic patients.

$\mathrm{TcPO}_{2}$ is a well-established tool to evaluate healing potential in diabetic patients. This test is 
able to reflect the microvascular status of the skin, measuring tissue perfusion. ${ }^{44}$

The literature scan retrieved just two pertinent articles. ${ }^{42,43}$ One described a heel ulcer in a young diabetic female wherein the dorsal ABPI and $\mathrm{TcPO}_{2}$ were normal but the readings in the hind foot showed an ischemic heel. ${ }^{42}$ The second was a retrospective cohort study on 191 diabetic patients, reporting a high frequency of normal $\mathrm{TcPO}_{2}$ measurement when performed on the dorsum of the foot, whereas the rear foot oximetry revealed peroneal and posterior tibial arteries ischemia. ${ }^{43}$

The jury strongly agreed on the use of $\mathrm{TcPO}_{2}$ as a reliable noninvasive test to assess the rear foot perfusion in patients with HPIs. This recommendation may potentially change the way of assessing the heel vascular perfusion when pressure injuries are present.

This is an important aspect to consider in the decision-making process before selecting therapeutic interventions, such as sharp debridement or use of moisture retaining dressings.

The panel acknowledges that $\mathrm{TcPO}_{2}$ can be an expensive and not always available device; however, its uses should be increasingly implemented in clinical practice when a HPI is found.

RECOMMENDATION 5: In patients presenting with one or more HPIs stages II, III, and IV and impaired vascular perfusion $(A B P I<0.5$ and toe pressure $<30 \mathrm{mmHg}$ and / or $\mathrm{TcPO}_{2}<40 \mathrm{mmHg}$ ), occlusive moisture retaining dressings must be avoided, to prevent amputations and death. Grade: Strongly recommended.

Occlusive moisture retaining dressing is a common option in treating HPIs. However, this treatment is affected by a significant risk of spreading infections or gangrene, particularly when chronic ischemia is present.

We pursued to establish suitable criteria to identify patients at high risk for adverse effects. In our search, three articles ${ }^{16,45,46}$ were relevant for this topic, but none of them provided solid evidence. Nevertheless, jurors strongly recommended against the use of moisture retaining dressings in presence of the abovementioned instrumental findings. Further clinical research is needed to validate the recommended thresholds. Anyway, healing potential must be always assessed before using advanced dressings.

Indeed, when the healing is not the goal, maintaining a dry environment should be preferred to prevent spreading infections such as necrotizing fasciitis, wet gangrene, or sepsis. However, evidence is lacking even on this specific topic.

\section{Management of heel pressure injuries stages I and II}

Text of recommendations and statements, notes, and voting results are listed in Table 1.

RECOMMENDATION 6: In diabetic and nondiabetic adult patients at risk of developing HPIs, a polyurethane foam should be applied to prevent skin damage. Grade: Weakly recommended.

The second area of the consensus conference focused on the management of HPIs stages I and II. During the preliminary discussion, the panel felt the need to address preventive strategies. In particular, more than one expert advocated the use of polyurethane foam dressing.

Evidence search identified many articles on this topic. The use of a five-layer heel-shaped foam dressing has shown the ability of preventing HPIs in critically ill patients in intensive care. ${ }^{47} \mathrm{~A}$ similar study showed different results in a geriatric facility, where no significant difference in incidence of HPIs was observed in comparison with the control group. ${ }^{48}$

Although further studies are needed to confirm the effectiveness of this type of dressings, the jury found this intervention advisable, with a weak degree of strength.

RECOMMENDATION 7: In diabetic and nondiabetic adult patients with existing HPIs stages I and II, a low friction technology (LFT) device must be applied to prevent further damage. Grade: Strongly recommended.

Existing HPIs need to be treated locally along with a proper offloading. ${ }^{49}$ This is a general rule and part of the standard practice. The panel was interested in understanding whether a LFT device should be recommended in all patients with existing low stage HPIs.

A NICE medical technology guidance ${ }^{26}$ identified by manual literature search, analyzed available data about LFT devices (boots or undergarments branded "Parafricta" ${ }^{\circledR}$ ).

The adoption of this device in clinical practice could reduce incidence of HPIs and must be adopted as part of standard care (strong agreement among jurors), both in patients deemed to be at risk of or with already existing HPIs. The reduction of friction coefficient appears, indeed, beneficial to prevent worsening of lesions.

RECOMMENDATION 8: In diabetic and nondiabetic adult patients, presenting with HPIs, a silicone dressing must be used to prevent medical adhesive-related skin injuries (MARSIs). Grade: Strongly recommended.

RECOMMENDATION 9: In neonates and pediatric population presenting with HPIs, a silicone 
dressing must be used to avoid MARSIs. Grade: Strongly recommended.

Interest about skin tears is rapidly increasing. In particular, MARSIs appear a relevant under-recognized but preventable problem. ${ }^{33}$ The panel created a specific clinical question on this topic either for adult/diabetic population or children/infants. Limited evidence suggests the use of silicone dressings to prevent MARSI ${ }^{33}$ due to its ability of maintaining a constant adherence over time, reducing the force needed to remove it. In particular, silicone is useful either as a primary dressing (silicone sheet) or as a main constituent for tapes and gentle borders. ${ }^{50-52}$

A strong agreement was reached for both populations.

\section{Management of HPIs stages III and IV, suspected deep tissue injuries, and injuries of unknown depth}

Text of recommendations and statements, notes, and voting results are listed in Table 1.

RECOMMENDATION 10: In adult patients presenting with one or more HPIs stage III with a normal limb perfusion and no signs of infection, the negative pressure wound therapy (NPWT) should be started to promote the healing process and reduce complications. Grade: Weakly recommended.

NPWT is considered a safe and effective treatment for different kinds of wounds. ${ }^{25}$ The panel suggests with a low level of agreement the use of NPWT as an effective treatment in stage III HPI not complicated with infection or poor vascular supply to speed up the healing process.

Moisture retaining dressings are widely recognized as part of standard practice for the treatment of pressure ulcers. However, the panel discussed about the potential role of adding a biophysical agent to improve and speed up the healing process of HPIs stage III, also avoiding further complications. In particular, experts substantially agreed to include NPWT as one of the options in not complicated stage III HPIs.

NPWT with instillation could be advisable even in presence of local infection. The panel underlined the need of skilled professionals. Moreover, a preliminary evaluation of the healing potential and life expectancy before using a treatment more expensive than standard treatment is strongly advisable. Established protocols could help clinicians in making the right choice.

RECOMMENDATION 11: In adult patients presenting with one or more HPIs stage IV, a surgical intervention must be performed to support the wound healing and prevent major amputation. Grade: Strongly recommended.
RECOMMENDATION 12: In adults presenting with one or more HPIs stage IV complicated with bone infection involving surrounding soft tissues, a surgical approach must be performed to support the wound healing and prevent major amputation. Grade: Strongly recommended.

Local treatment of HPIs is mainly empirical. In particular, evidence supporting surgical or conservative approach is completely lack. The poor available literature (a case series of three patients treated with surgical intervention ${ }^{10}$ and a narrative review on HPIs treatment ${ }^{13}$ ) underlines possibility to achieve a complete healing in patients with stage IV HPIs and the need of a surgical intervention to obtain wound repair.

A long debate with the experts led to include these two recommendations in favor of surgery for stage IV HPIs regardless of the presence of osteomyelitis. The most discussed items were life expectancy, vascular supply, extension of damage, and type of procedure such as partial and/or total calcanectomy or other more limited surgical interventions. Critical aspects as timing, techniques, and extension of surgical resection largely rely on empirical basis and require a thorough multidisciplinary discussion and transparent trade-off with patients' "values and preferences."

The jury reached a strong level of agreement for both recommendations.

RECOMMENDATION 13: In ambulatory diabetic and nondiabetic adult patients without vascular impairment, the panel cannot recommend in favor or against the removal of dry eschar eventually present on the heel to reduce the healing time, improve functional lower limb recovery, and reduce infectious risk and pain. Grade: Uncertain.

A dry and stable eschar on heel wound could protect the calcaneus, as suggested by European Pressure Ulcer Advisory Panel (EPUAP) guidelines. ${ }^{49}$ However, some clinicians believe that necrotic tissue should be always removed to improve healing process, particularly in diabetic patients. A specific clinical question was thoroughly discussed, but the jury did not reach enough agreement. This uncertainty may potentially have negative implications in clinical practice. As a consequence, this should be a high-priority topic for future research.

RECOMMENDATION 14: In diabetic and nondiabetic adult patients presenting with heel SDTI and blood blisters, fluid should be aspirated from blisters to improve the healing process and reduce the risk of infection and pain. Grade: Weakly recommended.

SDTI is defined as purple or maroon localized area of discolored intact skin or blood-filled blister 
due to damage of underlying soft tissue from pressure and/or shear. ${ }^{49}$ How to manage blisters is still not clear, and conflicting opinions affect health professionals' world. Although there is complete lack of evidence in this field, a weak agreement was achieved among our panelists on the usefulness of fluid aspiration of blood blisters, in particular for large lesions interfering with activity daily living and/or causing intense pain.

More recently, the sense of discussion moved through the optimal choice of treatment between aspiration and deroofing. This has been object of a randomized controlled trial, not conclusive in favor of one of these two procedures. ${ }^{53}$

Fluid aspiration from a blister requires skilled professionals and carries some risks (infections and bleedings).

RECOMMENDATION 15: In neonates and pediatric patients with yellow eschar, a conservative approach with moisture retaining dressings should be used to promote wound healing. Grade: Weakly recommended.

RECOMMENDATION 16: In neonates and pediatric patients with black stable eschar, a conservative approach based on a local disposable applicator containing a small amount of $2 \%$ gluconate chlorhexidine should be used to promote a progressive local dehydration and wound healing. Grade: Weakly recommended.

The sharp debridement of yellow eschar and dry black eschar in neonates and pediatric population can be counterproductive due to the low thickness of pediatric heel tissues and the impossibility to distinguish soft fat tissues from muscles. This point was explored together with the two pediatric plastic surgeons of the panel. A conservative approach with moisture retaining dressings seems preferable to promote wound healing in yellow eschar. In dry and stable black eschar, progressive local dehydration is a promising approach (weak agreement). ${ }^{54}$

In yellow eschar the deep damage is usually low. On the contrary, black eschar, as unstageable lesion, requires the use of ultrasound scan to assess the full thickness involvement. ${ }^{55}$

In a personal series of 15 cases (G. Ciprandi, unpublished data), all children affected by full thickness heel injuries showed a black eschar. Even if the literature search was not helpful on this topic and only two studies pertinent to black eschar treatment were retrieved, the prevalent opinion of the authors is that a conservative treatment, in these patients, is mandatory. ${ }^{56-58}$ Evidence of spontaneous healing of transient damages due to heel prick needle injuries is something supporting the choice of a conservative treatment for black eschar. ${ }^{59}$
RECOMMENDATION 17: In neonates and pediatric patients with infected HPIs any stage, the panel cannot recommend in favor or against an antimicrobial dressing to control the local bioburden. Grade: Uncertain.

The use of antimicrobials in neonates and pediatric patients requires caution. Iodine and silver sulfadiazine could be dangerous due to systemic absorption and related toxicity, especially in a long-term run. Nonmedicated technology dressings made with hydrophobic binding technique may be preferable for infection management in HPIs but many conflicting opinions arose during discussion.

The jury did not reach an agreement and, therefore, we could not recommend a definite clinical behavior. Further clinical trials are strongly needed to clarify this topic.

RECOMMENDATION 18: In neonates and pediatric patients with noninfected stages III and IV HPIs and residual defect, a dermal regeneration template (DRT) should be used to promote a faster healing. Grade: Weakly recommended.

The use of a DRT in HPIs and residual defect was discussed with the two plastic surgeons. The analysis of articles matching this clinical question did not reveal any study considering a dermal/ dermoepidermal substitute for residual defect in a newborn child with HPI. However, in pediatric plastic reconstructive surgery, a coverage of a skin soft tissue defect is well established. This approach provides a rapid healing, also reducing risk of local infection. ${ }^{60,61} \mathrm{~A}$ formal recommendation was formulated for this specific topic. The jury reached a level of agreement equal to weak.

\section{Offloading devices}

Text of recommendations and statements, notes, and voting results are listed in Table 1.

RECOMMENDATION 19: In all bedbound patients presenting with HPIs, an off-loading device with or without integrated wedge must be adopted to prevent further damage. Grade: Strongly recommended.

RECOMMENDATION 20: In paraplegic patients presenting with HPIs any stage, an off-loading device must be worn while sitting in wheelchair to prevent further damage and allow wound healing.

\section{Grade: Strongly recommended.}

RECOMMENDATION 21: Diabetic and nondiabetic adult patients presenting with HPIs stage IV-STDI or DU must avoid walking; an offloading device must be worn while on wheelchair, to prevent further damage and allow the wound healing. Grade: Strongly recommended. 
RECOMMENDATION 22: In diabetic and nondiabetic adult patients presenting with HPIs stages I and II, an off-loading device must be worn while walking to off-load the heels, prevent further damage, and allow the wound healing. Grade: Strongly recommended.

The steering committee created a specific area for offloading devices for patients with HPIs considering any stage, and both patients chair bound and bed bound with existing pressure ulcers. In particular, patients with HPIs stage IV-STDI or DU must avoid walking and an off-loading device must be worn while on wheelchair, whereas patients with stages I and II and stable stage III lesions can walk while wearing an off-loading device. See Table 1 for details about recommendations and notes on this area.

Although our experts and jurors strongly agreed on the need of offloading devices either in primary prevention or as part of any effective therapeutic strategy, widely discordant opinions emerged from the debate. In particular, podiatrists of our panel underlined the lack of specific criteria able to select a tailored offloading device.

Further studies should be undertaken to establish specific criteria supporting the choice of an offloading device in patients with heel pressure ulcers. This is a high-priority area for potential aftermaths on clinical and organizational outcomes.

\section{Biophysical agents and referral criteria}

Text of recommendations and statements, notes, and voting results are listed in Table 1.

RECOMMENDATION 23: In adult patients presenting with HPIs stages III and IV without vascular impairment, the NPWT delivering a negative pressure of $-75 /-125 \mathrm{mmHg}$ should be applied to promote the healing process. Grade: Weakly recommended.

RECOMMENDATION 24: In adult patients presenting with HPIs stages III and IV with mild vascular impairment $(A B P I>0.6$ to $<0.9)$, the NPWT delivering a negative pressure of $-75 /-125 \mathrm{mmHg}$ should be applied to promote the healing process. Grade: Weakly recommended.

RECOMMENDATION 25: In adult patients presenting with HPIs stages II and III without vascular impairment, the NPWT canister-free delivering a negative pressure of $-80 \mathrm{mmHg}$ should be applied to reduce the healing time. Grade: Weakly recommended.

STATEMENT 26: A "SIMPLE heel pressure injury" is defined as follows: a wound healable with conservative management along with proper off loading.
The diagnosis requires the presence of all following criteria:

1. First or second stage

2. Normal vascular perfusion

3. Not diabetic patient

4. Onset $<6$ weeks

Grade of agreement: Strong.

STATEMENT 27: A "COMPLEX heel pressure injury" is defined as follows: a wound unlikely to heal with a conservative management.

The diagnosis requires the presence of one or more of following criteria:

- Impaired vascular perfusion

- Third or fourth stage

- Unstageable (DU)

- Diabetic

- Onset $>6$ weeks

Grade of agreement: Strong.

STATEMENT 28: A "RECALCITRANT heel pressure injury" is defined as follows: a wound that is unlikely to heal despite a complete diagnostic workup and the standard treatment.

The diagnosis requires the presence of following criteria:

1. Static wound without signs of improvement for $>4-8$ weeks

\section{Grade of agreement: Strong.}

RECOMMENDATION 29: In all patients presenting with HPIs and positive probe-to-bone, a referral to a dedicated team must be done to rule out bone infections and tailor the right treatment to avoid further major complications like amputations. Grade: Strongly recommended.

STATEMENT 30: The introduction of an interdisciplinary heel pressure injury team for adults could improve HPI management, reduce major amputations, and support functional limb recovery. Grade of agreement: Strong.

STATEMENT 31: An interdisciplinary heel pressure injury team, essential members including at least:

- A surgeon/physician expert in wound care

- A specialist wound care nurse

- A podologist/podiatrist

improves heel pressure management, reduces major amputations and foot complications, and supports functional limb recovery.

Grade of agreement: Strong.

STATEMENT 32: An interdisciplinary heel pressure injury team for neonates and children could improve HPI management, reducing amputations 
and foot complications and supporting functional limb recovery. Grade of agreement: Strong.

RECOMMENDATION 33: In neonates and pediatric patients with unstageable HPIs, a threedimensional echography must be performed to assess the compromised deep tissue and underlying structures for further treatments. Grade: Strongly recommended.

RECOMMENDATION 34: In diabetic patients, presenting with one or more HPIs (any stage), an urgent referral to a diabetic foot center for a wound assessment must be obtained to reduce complications such as amputations and improve clinical outcomes, healing rate, and lower limb functional recovery. Grade: Strongly recommended.

The last set of recommendations and statements concerns "biophysical agents" and "referral criteria." The clinical decision of using biophysical agents, indeed, implies the referral of patients to specialist centers.

EPUAP guidelines ${ }^{49}$ suggest the use of a series of biophysical agents for nonhealing pressure ulcers, such as electroceutical therapy, pulsed radiofrequency electromagnetic field, and NPWT in adult patients.

Our panel decided to focus the discussion on NPWT only. NPWT could increase the healing rate of recalcitrant heel lesions, although this intervention is expensive, there are no protocols detailing rules for its effective and safe use, and skilled professionals are needed. Definite clinical pathways should be created to guide the use of NPWT in HPIs. So, we struggled to identify specific indications for clinical practice, achieving at least a weak agreement on NPWT use in stages III and IV HPIs, with or without vascular impairment, and in stage II HPIs without vascular impairment.

In this article, we did not provide specific indications on the wound bed preparation (WBP) prior NPWT application because these are beyond the scope of this project. However, clinicians should be fully aware that appropriate WBP, in accordance with time framework (tissue debridement, infection, or inflammation, moisture balance edge effect) is a fundamental pillar to adopt before using NPWT as any other wound healing approach. ${ }^{62,63}$

Moreover, NPWT can also be seen as an essential step in preparing wound bed. This tool could indeed accelerate the process when using a simple irrigation or a more specific instill procedure or simply being interspersed with brief repeated soft debridement, because of delicacy of this body area. Especially in patients who are more fragile, it appears to be a time-sparing and painless procedure. ${ }^{64,65}$
A safe NPWT application requires careful evaluation of specific conditions, such as bleeding risk, vascular anastomosis, necrotic wound bed, untreated osteomyelitis, neoplastic tissue, and others. ${ }^{34}$

International guidelines, such as EPUAP, points out that biophysical agents are specifically intended for "recalcitrant" lesions.

However, the definition of "recalcitrant" appears quite hazy. Therefore, the need of practical classification criteria is urgent to enhance the implementation of such recommendations in real world. Our panel, despite the lack of evidence, proposed statements 26-27-28 just aiming to stratify severity of clinical conditions, incorporating EPUAP point of view on pressure ulcers staging.

A strong agreement was achieved about three definitions for HPIs: SIMPLE, COMPLEX, and RECALCITRANT.

The use of these definitions could ease the decision-making process. For example, in a COMPLEX HPI, a local treatment is likely to be useless, posing more risks for the patient and for limb salvage. A more complex approach is required.

Moreover, the panel guesses that making this differentiation clear could improve patient satisfaction, safety, and rationale allocation of resources. In particular, adoption of clear definitions might help inexperienced professionals to understand better when seeking for help.

In contrast, the panel has often underlined that the availability of specific expertise is one of the major factors predicting good outcomes of patients with severe HPIs. Consequently, the creation of an interdisciplinary HPI team seemed a valid and strongly recommendable method to assure appropriate assistance to adult and pediatric patients. The jury endorsed this point of view with a strong grade of agreement, also providing suggestions for a minimum set of components. Anyway, similar structured approaches, based on specific guidelines, have been already proven useful in the care of diabetic ulcers.

Unfortunately, budget constraints could hinder or even prevent implementation of such interventions in real practice. In less severe cases, offloading could be effective in considerably improving the clinical picture and vascular referral could be preferable for heels characterized by predominant ischemia. Pilot studies aiming to verify cost/effectiveness of the adoption of these measures would be very important.

\section{FUTURE DIRECTIONS}

The heel is frequently affected by pressure wounds ${ }^{66-68}$ with a deep impact on relevant clinical outcomes including mortality. ${ }^{13}$ 
Despite that, our study confirms that very few and low-quality evidence from primary research, nor recommendations coming from guidelines able to inform clinical practice are available nowadays.

As a consequence, clinical diagnostic and therapeutic behaviors are characterized by wide heterogeneity and empirical approach with obvious and unacceptable fallout on patient outcomes and wasting of resources.

Paucity of reliable trials on this topic relies on intrinsic characteristics of HPIs, such as lack of validated diagnostic and classification criteria for complex wounds, the need of multidisciplinary and multiprofessional approach, clinical variability in course and outcome, controversies in methods for measuring end-points and other methodological barriers (i.e., difficulties in maintaining blinding, high risk of "trial effect," and so on).

A solid clinical expertise, therefore, represents the only reliable guide for clinical practice. However, many factors limit transferability of specialists' opinion and its use in defining the "good clinical practice." Different background (surgical, medical, nursing, podiatry, etc.), as well as specific case mix and even individual attitudes or interests, contributes to create a great heterogeneity in experts' approaches, which often reflects in nonsystematic reviews and other informal consensusbased documents.

Many of these flaws can be addressed by more systematic consensus development methods. Our project aimed to avoid bias among discussants, through the adoption of a rigid methodological frame and involvement of an international, largely representative, panel. Furthermore, formal consensus conferences allow reasons of controversies to become explicit, avoiding disagreements strictly related to misunderstandings over terms or definitions. Clear measure of grade of consent, identification of most urgent gray areas to propone for future research, and trading off of interests coming from different specialties and professions are other relevant benefits of these initiatives.

However, these projects require considerable efforts in terms of time, human resources, and economic burden, exposing them to potential conflicts of interests. In our study, small contributions coming from a high number of industries minimized the risk.

Moreover, high prevalence of HPIs, considerable potential severity, wide variability in clinical behaviors, and large impact on health systems' resources not only worth such relevant effort but also make the potential value added by our study truly great.

We believe that the results of our effort could improve outcomes, especially in areas such as vascular assessment, offloading devices, pressure ulcers classification, and referral criteria where clear and shared opinions emerged.

However, a number of barriers could hinder the application of recommendations. In particular, budget constraints, ingrained habits and beliefs as well as the need of a substantial upgrade in specific skills are among the main obstacles.

Surgical intervention for HPIs stage IV, for example, requires surgeons with expertise, together with a skilled staff, a resource not available in many clinical settings. An interdisciplinary team for heel pressure ulcers would be an effective tool to improve practice, but high costs and scattered distribution of expert professionals limit its implementation to few highly specialized hospitals.

Moreover, the potential impact of widespread application of systematically developed recommendations on relevant clinical outcomes, such as preserving lower limb and its functionality, should be better understood by policy makers and health care managers, to prevent unnecessary suffering and wasting money. Adequate organizational measures and budget plans should be adopted accordingly.

A number of methodological limits have to be pointed out. First, the extreme scarcity of evidence made the selection of topics, diagnostic and therapeutic interventions and outcomes, considered as alternative options, largely arbitrary. Then, methodological rigor itself and the choice to seek the broadest possible consensus could have hidden minority reports. Finally, potential implications in legal controversies cannot be neglected. In this regard, we want to clearly state that our recommendations are intended to support clinical practice mainly in typical clinical scenarios. Deviations from suggested behaviors could be requested by specific characteristics of patients or wounds.

More than $34 \%$ of recommendations and statements did not achieve a strong level of consensus. Among the reasons underlying this high rate of relatively poor agreement are the large number of jurors, their heterogeneous provenance and expertise, and the use of a two-round only Delphi method.

Active implementation of recommendations developed by our study will be an outstanding challenge for the next future. Previous experiences ${ }^{69}$ showed, indeed, negligible improvements in practice after the publication of expert-based recommendations, even when coming from authoritative institutions (e.g., NIH). Adequately funded strategies such as clinical audits are strongly needed to promote a meaningful improvement in real-world 
practice and to verify the impact on clinical and organizational outcomes.

\section{ACKNOWLEDGMENT AND FUNDING SOURCES}

The consensus conference was mainly funded by AISLeC. Some industries (Angelini farmaceutici, Coloplast, Convatec Italia, Paul Hartmann, Sofar, and Vega) gave a small financial support, which has been used to partially cover organizational costs and travel expenses of the consensus group members; funds were administered through AISLeC. None of the sponsors actively participated in any aspect of the consensus, nor did they provide any other kind of material support or exercise any editorial influence over the consensus statements. This article solely reflects the opinions of the participating experts.

\section{AUTHORS DISCLOSURE AND GHOSTWRITING}

Authors declare no competing financial interests. No ghostwriters were used to write this article.

\section{ABOUT THE AUTHORS}

Massimo Rivolo, RN, RGN, BSc (Hons), MSc Wound Care, is an independent tissue viability nurse consultant, trained lymphoedema specialist, past EWMA board member for cooperating organizations (AISLeC), and executive member of World Union of Wound Healing Societies (WUWHS), Turin, Italy. Sara Dionisi, RN, is a methodologist, currently performing a doctoral research fellowship at University of Bologna, Italy. Diletta Olivari, MD, is a methodologist, internal medicine resident at Univesrsità Politecnica delle Marche, Ancona, Italy. Guido Ciprandi, MD, is responsible for neonatal and pediatric wound care, UOC Plastic Surgery, Department of Surgery, Ospedale Bambin Gesù Roma, Professor of Wound Care University of Rome "La Sapienza." Serena Crucianelli, MD, is a plastic surgeon, pediatric and adults' wound care specialist, Ospedale Bambin Gesù, Roma. Silvia Marcadelli, RN, PhD, is a methodologist, AUSL Bologna, Italy. Rosarita Zortea, RN, is a former AISLeC president. Fabio Bellini, RN, MSc Wound Care, ATS Val Camonica, Sondrio, Italy. Matteo Martinato, RN, $\mathbf{P h D}$, is a methodologist at Azienda Ospedaliera di Padova, Italy. Armando Gabrielli, MD, is a methodologist and full professor in internal medicine at Università Politecnica delle Marche, Ancona, Italy. Giovanni Pomponio, MD, is a methodologist and internist at Ospedali Riuniti di Ancona, Italy.

\section{SUPPLEMENTARY MATERIAL}

Supplementary Table S1

Supplementary Table S2

Supplementary Figure S1

\section{REFERENCES}

1. VanGilder C, Lachenbruch C, Algrim-Boyle C Meyer $S$. The International Pressure Ulcer Prevalence $^{\mathrm{TM}}$ Survey: 2006-2015: a 10-year pressure injury prevalence and demographic trend analysis by care setting. J Wound Ostomy Continence Nurs 2017:44:20-28.

2. Bennett G, Dealey C, Posnett J. The cost of pressure ulcers in the UK. Age Ageing 2004;33 230-235.
3. Dealey C, Posnett J, Walker A. The cost of pressure ulcers in the United Kingdom. J Wound Care 2012;21:261, 262, 264, 266.

4. Fowler E, Scott-Williams S, McGuire JB. Practice recommendations for preventing heel pressure ulcers. Ostomy Wound Manage 2008;54:42-48, 50-52, 54-57.

5. Helvig El, Nichols LW. Use of high-frequency ultrasound to detect heel pressure injury in elders.
J Wound Ostomy Continence Nurs 2012;39:500508.

6. Van Gilder C, Lachenbruch C, Harrison P, Davis D. Overall Results from the 2011 International Pressure Ulcer Prevalence Survey. Proceedings from Wound + Ostomy and Continence Nursing Society's 44th Annual Conference, Charlotte, NC, 2012.

7. Thorpe L. Assessing preventing and managing heel pressure ulcers. Wounds UK 2017;13:67-70. 
8. Gefen A. The biomechanics of heel ulcers. J Tissue Viability 2010;19:124-131.

9. Ciprandi G, Crucianelli S. Top Tips: preventing pressure ulcers in premature babies and neonates. Wounds Int 2015;6:5-9.

10. Boffeli TJ, Collier RC. Near total calcanectomy with rotational flap closure of large decubitus heel ulcerations complicated by calcaneal osteomyelitis. J Foot Ankle Surg 2013;52:107-112.

11. Khoo R, Jansen S. Slow to heel: a literature review on the management of diabetic calcaneal ulceration. Int Wound J 2018;15:205-211.

12. Yang K, Graf A, Sanger J. Pressure ulcer reconstruction in patients with heterotopic ossification after spinal cord injury: a case series and review of literature. J Plast Reconstr Aesthet Surg 2017; 70:518-528.

13. Bosanquet DC, Wright AM, White RD, Williams IM. A review of the surgical management of heel pressure ulcers in the 21 st century. Int Wound $\mathrm{J}$ 2016;13:9-16.

14. Black J, Alves P, Brindle CT, et al. Use of wound dressings to enhance prevention of pressure ulcers caused by medical devices. Int Wound $\mathrm{J}$ 2015;12:322-327.

15. Salcido R, Lee A, Ahn C. Heel pressure ulcers: purple heel and deep tissue injury. Adv Skin Wound Care 2011;24:374-380.

16. Reddy M. Pressure ulcers. BMJ Clin Evid 2011;28: 1901.

17. Nair R, Aggarwal R, Khanna D. Methods of formal consensus in classification/diagnostic criteria and guideline development. Semin Arthritis Rheum 2011:41:95-105.

18. Higher Institure of Health. National Plan Guidelines [In Italian]. www.psy.it/wp-content/uploads/ 2018/02/Manuale-Metodologico-Consensus.pdf (last accessed August 2019).

19. Brown P, Brunnhuber K, Chalkidou K, et al. Hoe to formulate research recommendations. BMJ 2006; 333:804-806.

20. Grade Working Group. http://www.gradeworking group.org (last accessed August 2019).

21. Jaeschke, Guyatt GH, Dellinger $P$, et al. Use of GRADE grid to reach decisions on clinical practice guidelines when consensus is elusive. BMJ 2008; 337:a744.

22. Wong JKF, Amin K, Dumville JC. Reconstructive surgery for treating pressure ulcers. Cochrane Database Syst Rev 2016;12:CD012032.

23. Lam K, van Asten SA, Nguyen T, et al. Diagnostic Accuracy of Probe to Bone to Detect Osteomyelitis Influence of osteomyelitis location in the foot of diabetic patients with transtibial amputation. Clin Infect Dis 2016;63:944-948.

24. Dumville JC, Webster J, Evans D, Land L. Negative pressure wound therapy for treating pressure ulcers. Cochrane Database Syst Rev 2015;5:CD011334.
25. Vikatmaa $P$, Juutilainen $V$, Kuukasjärvi $P$, Malmivaara A. Negative pressure wound therapy: a systematic review on effectiveness and safety. Eur J Vasc Endovasc Surg 2008;36:438-448.

26. Parafricta Bootees and Undergarments to reduce skin breakdown in people with or at risk of pressure ulcers. NICE Medical technologies guidance [MTG20], 2014. nice.org.uk/guidance/ mtg20 (last accessed February 21, 2019).

27. Dinh MT, Abad CL, Safdar N. Diagnostic accuracy of the physical examination and imaging tests for osteomyelitis underlying diabetic foot ulcers: meta-analysis. Clin Infect Dis 2008;47:519-527.

28. Levy A, Gefen A. Computer modeling studies to assess whether a prophylactic dressing reduces the risk for deep tissue injury in the heels of supine patients with diabetes. Ostomy Wound Manage 2016;62:42-52.

29. Levy A, Frank MB, Gefen A. The biomechanical efficacy of dressings in preventing heel ulcers. J Tissue Viability 2015;24:1-11.

30. Lund C. Medical Adhesives in the NICU. Newborn Infant Nurs Rev 2014;14:160-165.

31. Hunt $\mathrm{S}$. Promoting skin safety within the community. Br J Community Nurs 2016;21(Suppl. 12):S36-S38.

32. Gupta S, Ichioka S. Optimal use of negative pressure wound therapy in treating pressure ulcers. Int Wound J 2012;9(Suppl. 1):8-16.

33. McNichol L, Lund C, Rosen T, Gray M. Medical adhesives and patient safety: state of the science: consensus statements for the assessment, prevention, and treatment of adhesive-related skin injuries. Orthop Nurs 2013;32:267-281.

34. Apelqvist J, Willy C, Fagerdahl AM, et al. EWMA document: negative pressure wound therapy. $J$ Wound Care 2017;26(Suppl. 3):S1-S154.

35. Crowell A, Meyr AJ. Accuracy of the ankle-brachial index in the assessment of arterial perfusion of heel pressure injuries. Wounds 2017;29:51-55.

36. Okuwa M, Sanada H, Sugama J, et al. A prospective cohort study of lower-extremity pressure ulcer risk among bedfast older adults. Adv Skin Wound Care 2006;19:391-397.

37. Twilley $\mathrm{H}$, Jones $\mathrm{S}$. Heel ulcers-pressure ulcers or symptoms of peripheral arterial disease? An exploratory matched case control study. J Tissue Viability 2016;25:150-156.

38. Masaki N, Sugama J, Okuwa M, et al. Heel blood flow during loading and off-loading in bedridden older adults with low and normal ankle-brachial pressure index: a quasi-experimental study. Biol Res Nurs 2013;15:285-291.

39. Halliday A, Bax JJ. The 2017 ESC Guidelines on the diagnosis and treatment of peripheral arterial diseases, in collaboration with the European Society for Vascular Surgery (ESVS). Eur J Vasc Endovasc Surg 2018;55:301-302.

40. Peripheral Arterial Disease: Diagnosis and Management Evidence review for determining diagnosis and severity of PAD in people with diabetes. NICE guideline CG147, 2012 (Updated 2018). http://nice .org.uk/guidance/cg147 (last accessed June 2019).

41. zShishehbor MH, Hammad TA, Zeller T, Baumgartner I, Scheinert D, Rocha-Singh KJ. An analysis of IN.PACT DEEP randomized trial on the limitations of the societal guidelines-recommended hemodynamic parameters to diagnose critical limb ischemia. J Vasc Surg 2016;63:1311-1317.

42. Faglia E, Clerici G, Caminiti M, Vincenzo C, Cetta $F$. Heel ulcer and blood flow: the importance of the angiosome concept. Int J low Extrem Wounds 2013;12:226-230.

43. Izzo V, Meloni M, Fabiano S, et al. Rearfoot transcutaneous oximetry is a useful tool to highlight ischemia of the heel. Cardiovasc Intervent Radiol 2017:40:120-124.

44. Fagher K, Katzman P, Löndahl M. Transcutaneous oxygen pressure as a predictor for short-term survival in patients with type 2 diabetes and foot ulcers: a comparison with ankle-brachial index and toe blood pressure. Acta Diabetol 2018;55:781-788.

45. Kammerlander G, Eberlein T. Use of Allevyn Heel in the management of heel ulcers. J Wound Care 2003;12:313-315.

46. Hampton S. An evaluation of a silicone adhesive shaped heel dressing. Br J Nurs 2010;19:\$30S33.

47. Santamaria N, Gerdtz M, Liu W, et al. Clinical effectiveness of a silicone foam dressing for the prevention of heel pressure ulcers in critically ill patients: border II Trial. J Wound Care 2015;24: 340-345.

48. Santamaria N, Gerdtz M, Kapp S, Wilson L, Gefen A. A randomised controlled trial of the clinical effectiveness of multi-layer silicone foam dressings for the prevention of pressure injuries in high-risk aged care residents: the Border III Trial. Int Wound J 2018;15:482-490.

49. EPUAP/NPUAP/PPPIA (National Pressure Ulcer Advisory Panel, European Pressure Ulcer Advisory Panel and Pan Injury Alliance). In: Haesler E, ed. Prevention and Treatment of Pressure Ulcers: Quick Reference Guide. Perth, Australia: Cambridge Pacific Pressure Media, 2014. https://www.npuap .org/wp-content/uploads/2014/08/Updated-10-1614-Quick-Reference-Guide-DIGITAL-NPUAP-EPUAPPPPIA-160ct2014.pdf (last accessed February 21, 2019).

50. King A, Stellar JJ, Blevins A, Shah KN. Dressings and products in pediatric wound care. Adv Wound Care (New Rochelle) 2014;3:324-334.

51. Grove GL, Zerweck CR, Ekholm BP, Smith GE, Koski NI. Randomized comparison of a silicone tape and a paper tape for gentleness in healthy children. J Wound Ostomy Continence Nurs 2014; 41:40-48.

52. Morris C, Emsley P, Marland E, Meuleneire F, White R. Use of wound dressings with soft silicone adhesive technology. Paediatr Nurs 2009;21:38-43.

53. Ro HS, Shin JY, Sabbagh MD, et al. Effectiveness of aspiration or deroofing for blister man- 
agement in patients with burns: a prospective randomized controlled trial. Medicine (Baltimore) 2018;97:e0563.

54. Bates-Jensen BM. Wound care nurses' judgements on healing time in chronic wounds. Ostomy Wound Manage 1996;42:36-38.

55. Honaker JS, Forston MR, Davis EA, Weisner MM, Morgan JA, Sacca E. The effect of adjunctive noncontact low frequency ultrasound on deep tissue pressure injury. Wound Repair Regen 2016 24:1081-1088.

56. Baharestani MM, Ratliff CR. Pressure ulcers in neonates and children: an NPUAP white paper. Adv Skin Wound Care 2007;20:208-220

57. Ciprandi G. Facing pressure ulcers in Childhood. Swedish J Wound Care 2014;6:23-27.

58. Freundlich K. Pressure injuries in medically complex children: a review. Children (Basel) 2017:4:E25

59. Goodwin D, Supachana N. A safety lancet for neonatal blood spot tests: a design that facilitates pain-free, atraumatic samples. Br J Nurs 2019;28: S24-S28

60. Yuan XG, Zhang X, Fu YX, et al. Sequentia therapy with "vacuum sealing drainage-artificial dermis implantation-thin partial thickness skin grafting" for deep and infected wound surfaces in children. Orthop Traumatol Surg Res 2016;102 369-373.

61. Ghazi BH, Williams JK. Use of Integra in complex pediatric wounds. Ann Plast Surg 2011;66:493496.

62. Schultz GS, Barillo DJ, Mozingo DW, Chin GA Wound bed preparation and a brief history of TIME. Int Wound J 2004;1:19-32.

63. Atkin L, Bucko Z, Montero EC, et al. Implementing TIMERS: the raise against hard-to-heal wounds. J Wound Care 2019;23(Suppl. 3a):S1-S50.

64. Boie B. Rapid wound bed preparation using NPWT with hypochlorous acid instillation. Ostomy Wound Manage 2018;64:10.

65. Egemen 0, Ozkaya 0, Ozturk MB, et al. Effective use of negative pressure wound therapy provides quick wound bed preparation and complete graft take in the management of chronic venous ulcers. Int Wound J 2012;9:199.

66. Gallagher P, Barry P, Hartigan I, McCluskey P, O'Connor K, O'Connor M. Prevalence of pressure ulcers in three university teaching hospitals in Ireland. J Tissue Viability 2008;17:103-109.

67. Jiang 0 , Li X, Ou X, et al. The incidence, risk factors and characteristics of pressure ulcers in hospitalized patients in China. Int J Clin Exp Pathol 2014:7:2587-2594.
68. Gunningberg L, Stotts NA, Idvall E. Hospitalacquired pressure ulcers in two Swedish County Councils: cross-sectional data as the foundation for future quality improvement. Int Wound J 2011; 8:465-473.

69. Olivieri J, Manfredi L, Postacchini L, et al. Consensus recommendations for improvement of unmet clinical needs - the example of chronic graftversus-host disease: a systematic review and meta-analysis. Lancet Haematol 2015;2:e297e305.

$$
\begin{aligned}
& \text { Abbreviations and Acronyms } \\
& \mathrm{ABPI}=\text { ankle-brachial pressure index } \\
& \mathrm{AISLeC}=\text { Italian nursing society for wound } \\
& \quad \text { care study } \\
& \mathrm{DRT}=\text { dermal regeneration template } \\
& \mathrm{DU}=\text { injuries of unknown depth } \\
& \mathrm{HPI}=\text { heel pressure injury } \\
& \mathrm{LFT}=\text { low friction technology } \\
& \mathrm{MARSI}=\text { medical adhesive-related skin injury } \\
& \mathrm{NIH}=\text { National Institute of Health } \\
& \mathrm{NPWT}=\text { negative pressure wound therapy } \\
& \mathrm{OHS}=\text { orphan heel syndrome } \\
& \mathrm{PTA}=\text { posterior tibial artery } \\
& \mathrm{SDTIS}=\text { suspected deep tissue injuries } \\
& \mathrm{TBPI}=\text { toe brachial pressure index } \\
& \mathrm{T} \mathrm{CPO}=\text { transcutaneous oxygen pressure } \\
& \mathrm{WBP}=\text { wound bed preparation }
\end{aligned}
$$

\section{Appendix}

\section{APPENDIX 1. ADVISORY PANEL}

Andrea Bellingeri, RN, IRCCS Policlinico San Matteo, Pavia-Fabio Bellini, RN, Unimed SrL-Centro Medico, Boario Terme BS-Giacomo Clerici, MD, Istituto di Cura "Città di Pavia," Pavia-Matteo Martinato, $\mathrm{RN}, \mathrm{PhD}$, Azienda Ospedaliera di Padova, PadovaAnnalisa Moscatelli, RN, ASST Ovest Milanese, Legnano, MI-Battistino Paggi, RN, NovaraGiovanni Pomponio, MD, Azienda Ospedaliero Universitaria Ospedali Riuniti, Ancona-Claudio Quartero, RN, Opera Don Orione-Istituto Camaldoli, Genova-Massimo Rivolo, RN, MSc Wound Care, Italy-Silvia Scaffidi Domianello, RN, MSc, Padova-Fabrizia Toscanella, MD, Policlinico Luigi Di Liegro, Roma-Rosa Rita Zortea, RN, Turriaco GO.

\section{APPENDIX 2. EXPERT PANEL}

Balduzzi Gianmario, Podiatrist, ASST Ovest Milanese. Centro Servizi Medici San Carlo, Chiari Abbiategrasso-Baracchi Elisabetta, RN, AO San Carlo Borromeo, MI-Bellingeri Andrea, RN, IRCCS Policlinico San Matteo, Pavia-Bellini Fabio, RN, Unimed SrL-Centro Medico, Boario
Terme BS, Bizzini Rina, RN, AUSL Bologna, BOBrambilla Roberto, MD, Ospedale Clinici Zucchi, Monza, MB-Ciprandi Guido, MD, Ospedale Pediatrico Bambin Gesu', Roma, RM-Clementi Alessandra, RN, ASST Ovest Milanese, Legnano, MI-Crucianelli Serena, MD, Ospedale Pediatrico Bambin Gesu', Roma, RM-Dionisi Sara, RN, AUSL Bologna, BO-Falciani Francesca, RN, Azienda USL Toscana Centro, Firenze, FI-Granara Debora, RN, ASL 3 Genovese, Genova, GEGreco Alessandro, MD, ASL Frosinone, Frosinone, FR-Guidi Valentina, RN, Azienda Ospedalierouniversitaria Ospedali Riuniti, Trieste TS-Marcadelli Silvia, RN, PhD, AUSL Bologna, Bologna, BO-Martinato Matteo, RN, $\mathrm{PhD}$, Azienda Ospedaliera di Padova, Padova, PD-Olivari Diletta, MD, Clinica Medica, Università Politecnica delle Marche, Ancona AN-Papa Giovanni, MD, Azienda Sanitaria Universitaria Integrata di Trieste, Trieste, TS-Pomponio Giovanni, MD, Clinica Medica, Ospedali Riuniti di Ancona, AN-Quartero Claudio, RN, Piccolo Cottolengo di Don Orione, Genova, GE-Rivolo Massimo, independent tissue viability nurse consultant, Turin, TO-Scaffidi Domianello Silvia, RN, Azienda ULSS 6 Euganea di Padova, Padova, PD—Speese Katia, RN, 
Ospedale Santa Maria del Carmine, Rovereto, TNTeobaldi Ilaria, Podiatrist, Azienda Ospedaliera Universitaria Integrata di Verona, Verona, VRToma Luigi, MD, IRCCS, "Regina Elena" e "San Gallicano," Roma, RM-Toscanella Fabrizia, MD, Policlinico Ospedaliero Luigi di Liegro, Roma, RMTurrini Mattia, RN, ASST Settelaghi, Varese, VA-Volpini Maurizio, Podiatrist, Podologia Volpini, Frascati, RM-Zortea Rosa Rita, RN, Turriaco GO.

\section{APPENDIX 3. JURY PANEL}

Cecchetto Maria Grazia, RN, Azienda Ospedaliera Padova, Padova, PD-Chadwick Paul, National Clinical Director College of Podiatry, Manchester, UK-Ciprandi Guido, MD, Ospedale Pediatrico Bambino Gesu', Roma, RM-Cutting Keith, RN, clinical research consultant, Hertfordshire, UK-Falciani Francesca, RN, Azienda USL Toscana Centro, Firenze, FI-Ge- thin Georgina, RN, Head of School of Nursing and Midwifery, Aras Moyola, IRL-Granara Deborah, RN, ASL 3 Genovese, Genova, GEPapa Giovanni, MD, Azienda Sanitaria Universitaria Integrata di Trieste, Trieste, TSPiaggesi Alberto, MD, Azienda OspedalieroUniversitaria Pisana, Pisa, PI-Probst Sebastian, PROF. Haute Ecole De Sante'-HES-SO Geneve, Geneve, CH-Teobaldi Ilaria, Podiatrist, Azienda Ospedaliera Universitaria Integrata di Verona, Verona, VR-Toma Luigi, MD, IRCCS, "Regina Elena" e "San Gallicano," Roma, RM-Jose' Verdu' Soriano, RN, MSc Nurse, $\mathrm{PhD}$. Profesor Titular de Universidad, Alicante, Spain-Karen Staines, RN, Director of Education and Research, Accelerate CIC, London, UK-Crucianelli Serena, MD, Ospedale Pediatrico Bambin Gesu', Roma, RM-Balduzzi Gianmario, Podiatrist, ASST Ovest Milanese. Centro Servizi Medici San Carlo, Chiari, Abbiategrasso, MI. 